\title{
LOAD FREQUENCY CONTROL OF HVDC LINK INTERCONNECTED POWER SYSTEM USING GENETIC ALGORITHM
}

\author{
Saurabh Chanana ${ }^{\bowtie}$, Saurabh Kumar \\ Department of Electrical Engineering, National Institute of Technology Kurukshetra, India \\ saurabh@nitkkr.ac.in
}

\begin{abstract}
Advances in power electronics have improved grid support functions such as tieline power control and frequency control, making renewable generation and High Voltage DC (HVDC) links more common in power system applications. Load Frequency Control (LFC) systems handle the complex interactions between the distributed generator and the control area with the HVDC link. In this work, LFC of a two-zone system including parallel AC/DC transmission links has been analysed. The parameters of this system are optimised using advanced genetic algorithm resulting in improved performance of system in terms of reduction in peak overshoots and settling time of frequency measurement, tie line power flow and area control error signals in an interconnected power system. The advantage of having parallel HVDC links is also demonstrated when performance is compared to system interconnected with only AC tie line.
\end{abstract}

Keywords: frequency measurement, automatic generation control, load frequency

Received: 30 April 2019 Accepted: 31 May 2019 control, HVDC interconnected system, tie line power, area control error.

\section{Introduction}

It is well known that the number of long-distance high capacity power exchanges between managed areas has increased significantly. The latest advances in integration of super grid and renewable energy interconnection in modern power systems have raised concerns about HVDC transmission, which is a new challenge for future power systems. This is due to the deregulation of the energy industry and the introduction of devices based on power electronics in the power grid [1]. The advantages of the HVDC system are to interconnect asynchronous networks and to transmit higher power over longer distances. These benefits have maintained interest for HVDC technology in industry and academia.

High voltage DC systems can also increase the reliability of large-scale interconnected system [2,3]. Communication, coordination, frequency control, and tie-line power control of the interconnected multi-zone system pose additional challenges in order to extend the application of HVDC control to interconnect system with wide area [4]. The interconnect power system has a plurality of control zones. Any mismatch between the demand and the generated power can cause a deviation of the link power flow or system frequency from their predetermined values. In order to eliminate deviations from each area, LFC is used to control the set points of the different generating units in these areas. Thus, the function of the LFC is to adjust the power generation from the various sources in each region so that the tie-line power and power system frequency are maintained within specified limits [5].

This paper is based on the use of previous concepts and traditional models, taking into account the new DC/AC combined scenario, and applications of DC interconnection to renewable energy system dissemination [8]. Power transmission and control has been improved by using the LFC scheme with existing transmission lines in parallel configuration, to function them as a DC-AC parallel link that interconnects two-control area of power system $[6,7]$. In the literature, to interconnect the LFC of a two-zone power system, DC/AC parallel links are used. LFC is implemented with various control schemes to improve the dynamic response. These systems are suitable for responding to the essential changes in the state of modern power systems. It is noteworthy that the LFC function can be used not only, for modern HVDC transmission and long-term dynamic control of Flexible AC Transmission System (FACTS) but also equipment for emergency control of frequency stability analysis $[10,11]$.

As mentioned, grids are in high demand for related issues such as increasing long-distance transmission, high-capacity transmission, integrating large-scale renewable energy with multi-area interconnect systems reliably, and have greater adaptability and control over the power flow of transmission systems. Tapping remote energy far from the consumer centre is driving force behind development of new HVDC transmission 
lines. Challenge usually involves the integration of solar energy, or integration of large offshore wind farms far from the coast. In these circumstances, DC transmission frames are actually considered to be advantageous and cheaper than AC [12]. In addition, power market consolidation is another driving force behind this new transmission line concept, which implies long-distance power transactions.

The fundamental challenge of power systems under separation is to develop communications and controllers to the appropriate level of operation and control of the various systems. Communication systems that is used to control the power systems operations leads to unwanted delays. Time delay system uses the LFC scheme for a given communication channel characteristic. Maximum delay is calculated to gain the knowledge about the stability analysis and control design [13]. Recently, time delays in load frequency controller design were considered in remote case studies. At the same time, there is not much to consider in case of complex DC/AC interconnection and LFC loop communication retransmissions [13,14]. A major application of HVDC transmission lines is that these lines can be operated in parallel with existing AC transmission lines and are designed to improve controllability. Therefore, they can be regarded as DC/AC parallel links connecting any two control areas. DC/AC parallel links are used in the literature to interconnect LFCs of two-zone power systems using various control methods to achieve a satisfactory dynamic response. However, none of them consider the complete DC link model for LFC applications. This feature is integrated into the DC link.

A Supplementary Power Modulation Controller (SPMC) is used to control power flow through the DC Link [11]. This controller is displayed during LFC operation. In this controller we are taking three signal frequency in area $\mathrm{i}$, area $\mathrm{j}$, and ac tie line power and given to the HVDC transfer function which is given in Fig 1 . It is necessary to propose more detailed HVDC station dynamic model for LFC research as the application and importance of HVDC link is increasing [14-18].

\section{Two-Area AC/DC Interconnected System}

The Load frequency control issues are discussed in detail in the literature on power system control [1-4]. For implementing LFC in a multi-area system, the concept of Area Control Error (ACE) was introduced. In the dual regional power system model, the ACE for the ith region is given by below equation.

$$
\mathrm{ACE}_{i}=\beta_{i} \Delta f_{i}+\Delta P_{i j}
$$

Where $f$ is the systems frequency $\Delta P_{i j}$ is the total tie-line power flow between two deferent areas $\left(\Delta P_{i j}=-\Delta P_{i j}\right) . \beta_{i}$ is the frequency bias, it is usually referred to the tie-line bias control. The state space representation of $i$ th area can be given as follows:

$$
\begin{gathered}
\Delta w_{i}=\frac{K_{p i}}{1+s T_{p i}}+\left[\Delta P_{m i}-\Delta P_{L i}-\Delta P_{t i e A C}\right], \\
\Delta P_{m i}=\sum_{k=1}^{n} \Delta P_{m, i k},
\end{gathered}
$$

where local load deviation for $i=1,2$ is represented by $\Delta P_{L i}$ and $\Delta P_{m, i k}(i=1,2)$ is output of generation company (GENCO) $k$ in area $i$. Power system gain and time constant is $K_{p i}$ and $T_{p i}$ respectively. Power system gain can be given by:

$$
K_{p i}=\frac{1}{D_{\text {sys }-i}}, T_{p i}=\frac{M_{\text {sys }-i}}{D_{\text {sys-i }}}
$$

where $D_{s y s-i}$ and $M_{\text {sys-i }}$ are damping and inertia constants respectively. The other variables defined as follows:

$$
\Delta P_{m, i k}=\frac{1}{1+s T_{t g, i k}}\left[\frac{1}{2 \pi R_{k}}-K_{l i} \Delta P_{r e f i}\right]
$$

Where $T_{t g}$ the total time constants of governor and turbine in each generator and which is equal to $\left(T_{t i k}+T_{g i k}\right)[5]$ and $R_{k}$ is droop characteristics for each GENCO. The reference power of generation in $i$ th area will be

$$
\Delta P_{r e f i}=\frac{A C E_{i}}{s}=\frac{1}{s}\left[\frac{\beta_{i}}{2 \pi} \Delta w_{i}+\Delta P_{t i e A C}\right]
$$




$$
\Delta P_{t i e A C}=\frac{T_{i j}}{s}\left[\Delta w_{i}-\Delta w_{j}\right],
$$

where $T_{i j}$ is synchronization power coefficient of $\mathrm{AC}$ tie line connecting area $i$ to $j$

$$
\mathrm{X}_{\mathrm{DC}}=\mathrm{K}_{\mathrm{fi}} \Delta w_{i}+K_{A C} \Delta P_{t i e A C}+K_{f j} \Delta w_{j},
$$

where $\mathrm{X}_{\mathrm{DC}}$ is the dc link control signal, $\mathrm{K}_{\mathrm{fi}}, K_{A C}$ and $K_{f j}$ are control gain so DC tie line power written as:

$$
\Delta P_{D C}=G_{p} X_{D C}
$$

here $G_{p}$ is HVDC link transfer function which is equal to

$$
\mathrm{G}_{\mathrm{p}}=\frac{1}{1+\mathrm{sT}_{\mathrm{DC}}} .
$$

The actual DC power flowing through the system gives the time constant of the HVDC unit. Due to the frequency offset of each interconnection area and the AC link power deviation (for any parallel AC line with an HVDC transmission link) the total cable power in AC/DC system will be:

$$
\Delta P_{i j}=\Delta P_{D C}+\Delta P_{t i e A C} .
$$

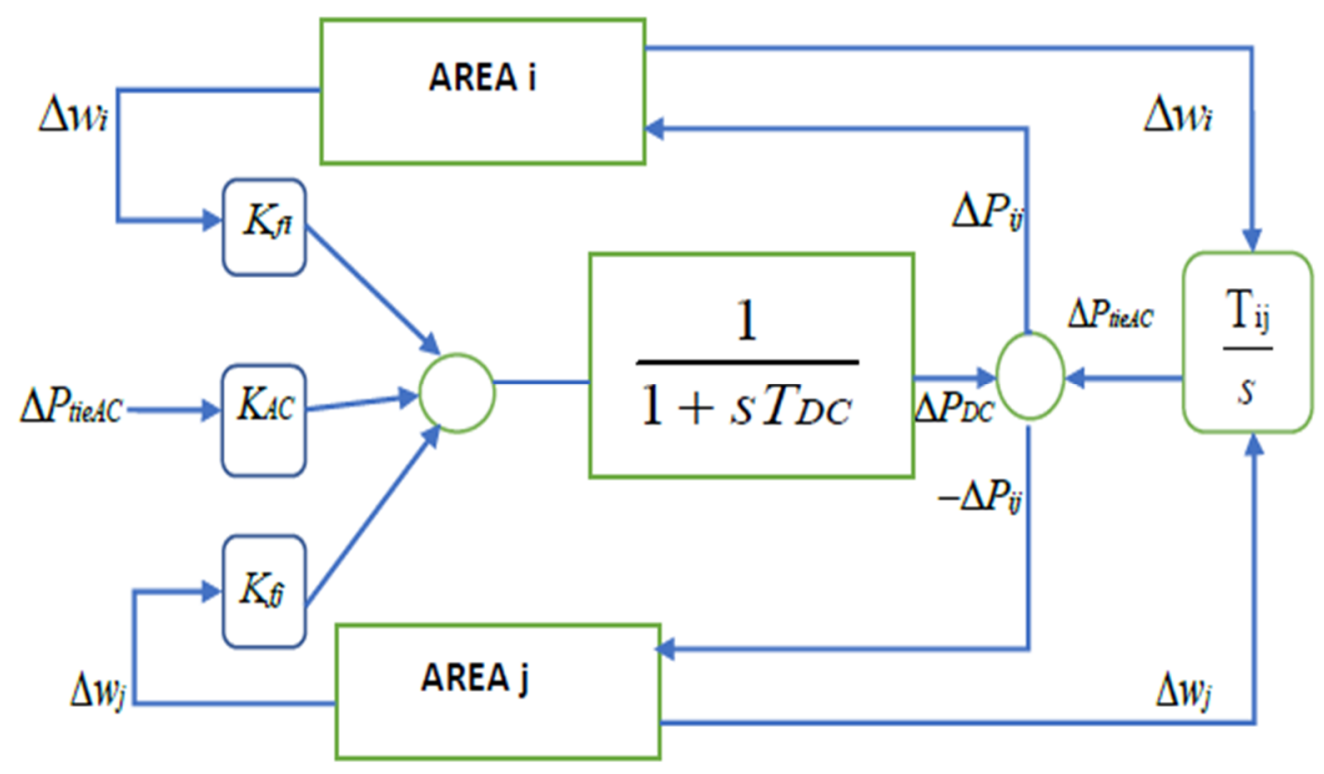

Figure 1: Model of SPMC for HVDC link interconnected system

\section{Optimization of LFC Parameters}

In the model of a two-area parallel DC/AC interconnected system shown in Fig. 2 there are three controllers which need to coordinate to give a desirable result that is stabilization of grid frequency and normalcy in tie line power flows. There is a requirement to tune the integral controller gains if respective areas $K_{I i}$ and $K_{I j}$. Further the three gains in SPMC viz. $K_{f 1}, K_{f 2}$ and $K_{A C}$ also need to set at value to give optimal response. In this work we have utilized the Genetic Algorithm to optimize these five parameters.

Genetic Algorithm (GA) is a metaheuristic developed by Holland in 1960s. It is inspired by the method of selection in nature. The decision variables to be found are binary coded in the form of strings of $0 \mathrm{~s}$ and $1 \mathrm{~s}$. The candidate solution is having a set of properties which can be altered. The starting point of evolution is a population having randomly generated individuals. GA is an iterative process, where the set of candidate solutions in each iteration called a generation. In one iteration, the fitness of individuals in the population is evaluated which usually amounts to the value of the objective function in the optimization problem being solved. The fit individuals are randomly selected from the current generation, and each individual's properties are modified (recombined and possibly randomly mutated) to form a new generation. The next iteration uses 
these new generation of candidate solutions. Usually, the algorithm terminates when either a maximum number of generations has been produced or no further improvement is achieved in the fitness function.

In this work, the integral of the squares of errors (ISE) for the three controllers in the system is taken as the fitness function.

$$
\mathrm{ISE}=\int_{0}^{\mathrm{t}_{\mathrm{min}}}\left[\Delta f_{1}^{2}+\Delta f_{2}^{2}+\Delta P_{\text {tieline }}^{2}\right]
$$

The steps of genetic algorithm used for finding optimal control parameters in this problem are underlined below.

\section{Step 1: Initialisation.}

The algorithm begins by creating a random initial population. In this work, the initial population contains 20 individuals.

\section{Step 2: Creating Next Generation.}

At each step, the algorithm uses the individuals in the current generation to create the next population. To create the new population, the algorithm performs the following steps.

- Scores each member of the current population by computing its fitness value as given above. These values are called the raw fitness scores.

- Scales the raw fitness scores to convert them into a more usable range of values. These scaled values are called expectation values.

- $\quad$ Selects members, called parents, based on their expectation.

- Some of the individuals in the current population that have lower fitness are chosen as elite. These elite individuals are passed to the next population.

- $\quad$ Produces children from the parents. Children are produced either by making random changes to a single parent - mutation - or by combining the vector entries of a pair of parents - crossover.

- Replaces the current population with the children to form the next generation. This type of replay is performed randomly in different places. As a result, new chromosome groups are generated in which the selection process can be resumed again.

\section{Step 3: Stopping Criterion.}

The algorithm stops when one of the stopping criteria is met. The genetic algorithm uses the following conditions to determine when to stop:

- Generations: The algorithm stops when the number of generations reaches the value of Generations.

- Time limit: The algorithm stops after running for an amount of time in seconds equal to Time limit.

- Fitness limit: The algorithm stops when the value of the fitness function for the best point in the current population is less than or equal to Fitness limit.

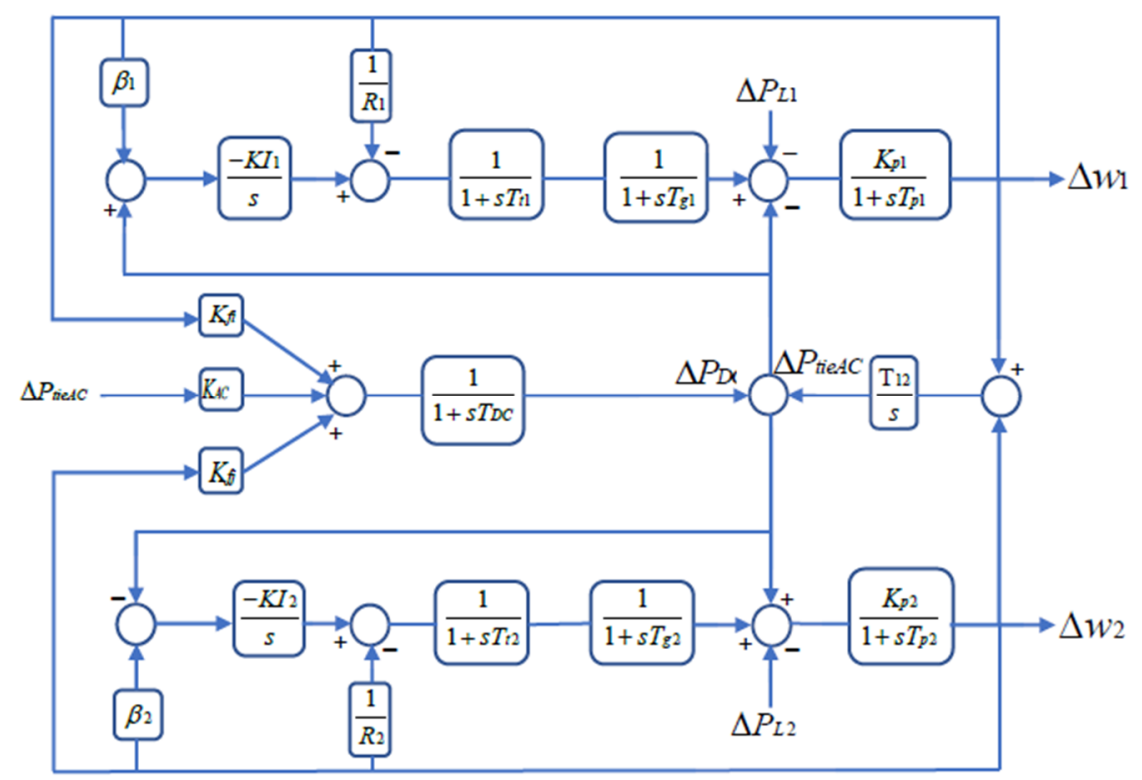

Figure 2: Two-area control model for HVDC link interconnected system with SPMC 


\section{Simulation Results}

The layout of two area power system considered in this study is shown in Fig. 3. Data sheet of this HVDC link interconnected systems given in Table 1 and 2.

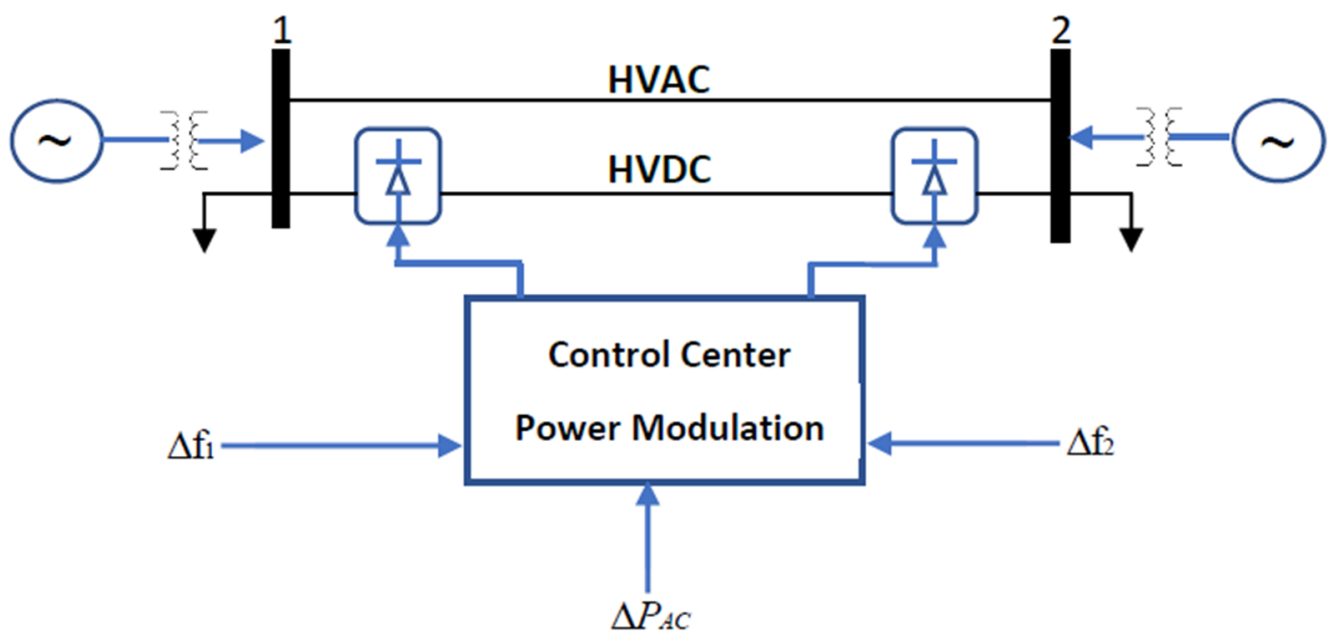

Figure 3: The layout of two area power system with HVDC link

Table 1: Parameters of GENCOs

\begin{tabular}{lll}
\hline Parameters & Area 1 & Area 2 \\
\hline$T_{g}(\mathrm{~s})$ & 0.06 & 0.06 \\
$T_{t}(\mathrm{~s})$ & 0.32 & 0.30 \\
$R(\mathrm{~Hz} / \mathrm{p} \cdot \mathrm{u})$ & 2.4 & 2.5 \\
\hline
\end{tabular}

Table 2: Control area parameters

\begin{tabular}{lll}
\hline Parameters & Area 1 & Area 2 \\
\hline$T_{p}(\mathrm{~s})$ & 20 & 25 \\
$K_{p}(\mathrm{~Hz} / \mathrm{p} \cdot \mathrm{u})$ & 102 & 102 \\
$\beta(\mathrm{p} \cdot \mathrm{u} / \mathrm{Hz})$ & .425 & .39 \\
$T_{12}(\mathrm{p} \cdot \mathrm{u} / \mathrm{Hz})$ & .245 & \\
\hline
\end{tabular}

This paper considers three cases for simulation studies to examine the performance of AC system, AC/DC system with standard controller gains [11] and AC/DC system in which parameters are optimized by genetic algorithm:

- $\quad$ Case-I; It is the case when we connect only AC tie line in the system.

- $\quad$ Case-II; It is the case when we use AC/DC system and we are taking standard controller parameters [11].

- Case-III; It is the case when we use AC/DC system and in this control, parameters are optimized by genetic algorithm, which is given by Table 3 . 
Table 3: GA optimized control parameters

\begin{tabular}{clll}
\hline Parameter & Minimum Bound & Maximum bound & Optimum Value \\
\hline$K_{f 1}$ & -5 & 5 & 1.712 \\
$K_{f 2}$ & -5 & 5 & -0.711 \\
$K_{A C}$ & -20 & 20 & 10.48 \\
$K_{I 1}$ & -10 & 10 & -5.379 \\
$K_{I 2}$ & -10 & 10 & -0.126 \\
\hline
\end{tabular}

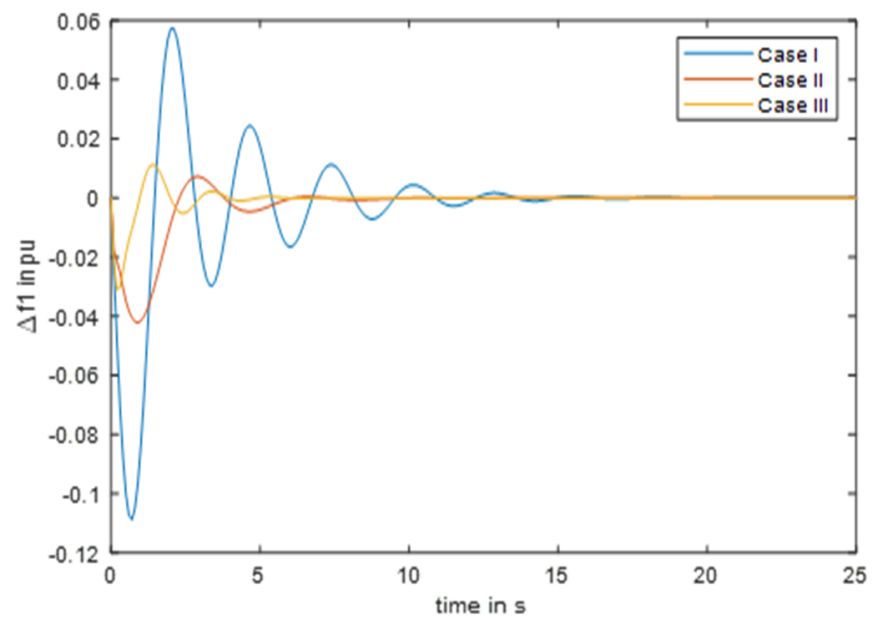

Figure 4: Frequency Deviation in Area 1

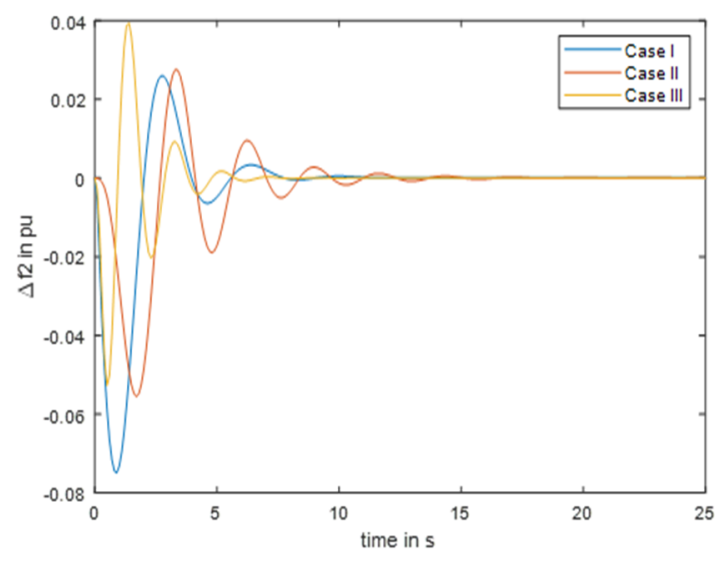

Figure 5: Frequency Deviation in Area 2

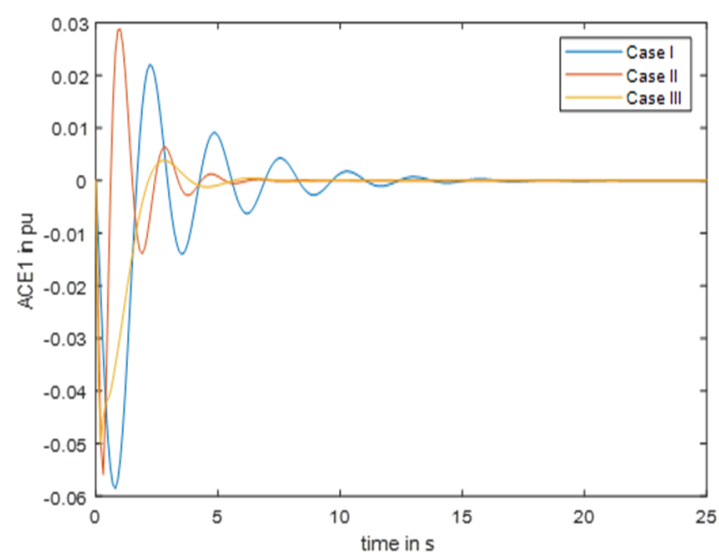

Figure 7: Area Control Error in Area 1

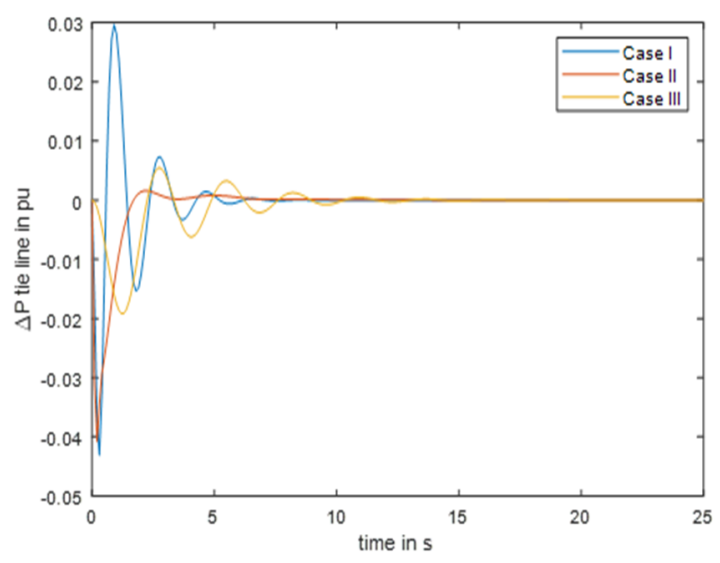

Figure 6: Tie Line Power Deviation

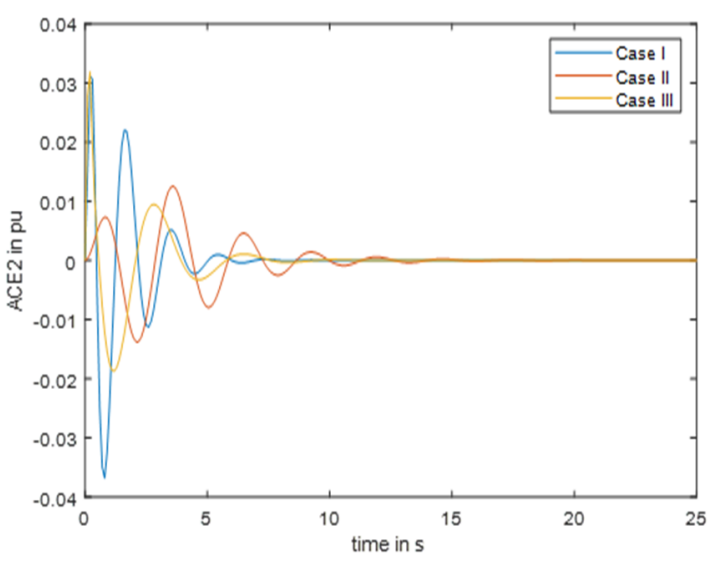

Figure 8: Area Control Error in Area 2 
Table 4: Overshoot (OS) [in p·u] and Settling Time (ST) [in s] of system response for different systems

\begin{tabular}{lccccccccccccc}
\hline & \multicolumn{2}{c}{$\Delta f_{1}$} & \multicolumn{2}{c}{$\Delta f_{2}$} & \multicolumn{4}{c}{$\Delta P_{\text {tie }}$} & \multicolumn{2}{c}{$\mathrm{ACE}_{1}$} & \multicolumn{2}{c}{$\mathrm{ACE}_{2}$} & \multicolumn{1}{c}{$\boldsymbol{I S E}$} \\
\hline & OS & ST & OS & ST & OS & ST & OS & ST & OS & ST & \\
CASE I & -0.108 & 15 & -0.074 & 11 & -0.043 & 7 & -0.586 & 14 & -0.013 & 8 & 0.1634 \\
CASE II & -0.042 & 7 & -0.055 & 13 & -0.041 & 14 & -0.559 & 7.5 & -0.018 & 12.5 & 0.0867 \\
CASE III & -0.031 & 5 & -0.052 & 6 & -0.020 & 12 & -0.05 & 7 & -0.036 & 6.5 & 0.035 \\
\hline
\end{tabular}

A comparison of response obtained from system in all three case has been depicted in Fig. 4 and 5 shown in deviation in frequency of area 1 and area 2 respectively for $5 \%$ deviation in load. Fig 6 shown the deviation in AC tie line power in control area 1 and area 2. From the Fig it is obtain that in Case II we get improved response as compared to Case I when HVDC interconnection is not utilize. This shown the utilities of parallel HVDC interconnection in LFC of 2 area system. In case III when control parameters of SPMC and LFC are optimized using genetic algorithm a further improvement in response is observed. These improvements are better utilized therefore data seen in table IV which show peak overshoot (in p·u), settling time (in s) for frequency error, tie line power error and area control error in both areas. The last column also shown the improvement in performance index ISE. The ISE reduce from 0.1634 in Case I to 0.0861 in Case II and further to 0.035 in Case III which shows considerable improvement in performance index.

\section{Conclusion}

This paper proposes an improved control strategy for multi-region interconnection linked through parallel AC/DC Link. The LFC of interconnected power system considering detailed dynamic models of HVDC links is presented due to its importance in future modern power systems. These simulation results distinguish between AC only interconnected systems and AC/DC interconnect systems. Using HVDC interconnect system, frequency errors, Tie line power deviations and ACEs have shorter settling times and smaller overshoots than traditional systems. Further the LFC and SPMC parameter are optimized using GA resulting in significant improvement over performance reported in literature earlier.

\section{References}

[1] Bevrani, H. and Hiyama, T. 2014. Intelligent automatic generation control. 2nd ed. CRC Press, Boca Raton, Florida, United States.

[2] Al-Haiki, Z. E. and Shaikh-Nasser, A. N. 2001. Power transmission to distant offshore facilities. IEEE Trans Ind Appl 47, 3, pp. 1180-1183.

[3] Giddani, O. A., Abbas, A. Y. M., Adam, G. P., Anaya-Lara, O., and Lo, K. L. 2013. Multi-task control for VSC-HVDC power and frequency control. Electric Power Energy Syst 53 ,pp. 684-690.

[4] Kundur, P. 1994. Power system stability and control. McGraw-Hill, New York, United States.

[5] Rakhshani, E. and Rodriguez, P. 2014. Active power and frequency control considering large scale RES. In Large scale renewable power generation. Springer-Verlag, Berlin, Heidelberg, pp. $233-271$. DOI: $10.1007 / 978-981-4585-30-9 \_9$

[6] Arya, Y. and Kumar, N. 2016. AGC of a multi-area multi-source hydrothermal power system interconnected via AC/DC parallel links under deregulated environment. Int J Electr Power Energy Syst 75 , pp. $127-138$.

[7] Donde, V., Pai, A., and Hiskens, I. A. 2001. Simulation and optimization in an AGC system after deregulation. IEEE Trans Power Syst 16, 3, pp. 481-489.

[8] Datta, M., Senjyu, T., Yona, A., Funabashi, T., and Chul-Hwan, K. 2011. A frequency-control approach by photovoltaic generator in a PV-diesel hybrid power system. IEEE Trans Energy Convers 26, pp. 559-571.

[9] Pourmousavi, S. A. and Nehrir, M. H. 2014. Introducing dynamic demand response in the LFC model. IEEE Trans Power Syst 29, 4, pp. 1562-1572.

[10] Shashi, K. P., Soumya, R. M., and Nand, K. 2013. A literature survey on load frequency control for conventional and distribution generation power systems. Renew Sustain Energy Rev 25, pp. 318-334.

[11] Rakhshani E., Remon, D., and Rodriguez, P. 2016. Effects of PLL and frequency measurements on LFC problem in multi-area HVDC interconnected systems. International Journal of Electrical Power 63 Energy Systems 81, October 2016, pp. 140-152. 
[12] Wolff, G. 2010. Supergrid costs and benefits, DESERTEC-UK. http://www.trecuk.org.uk/ resources/supergrid_costs1.pdf

[13] Sahin, S., Saffet, A., and Chika, O. N. 2015. An exact method for computing delay margin for stability of load frequency control systems with constant communication delays. IEEE Trans Power Syst 31, 1, pp. 370-377.

[14] Peng, C. and Zhang, J. 2015. Delay-distribution-dependent load frequency control of power systems with probabilistic interval delays. IEEE Trans Power Syst 31, 4, pp. 3309-3317.

[15] Du, Z. B., Zhang, Y., Liu, L., Guan, X. H., Ni, Y. X., and Wu, F. F. 2007. Structure-preserved power frequency slow dynamics simulation of interconnected ac/dc power systems with AGC consideration. IET Gener Transm Distrib 1, 6, pp. 920-927.

[16] Ganapathy, S. and Velusami, S. 2010. MOEA based design of decentralized controllers for LFC of interconnected power systems with nonlinearities, AC-DC parallel tie lines and SMES units. Energy Convers Manage 51, pp. 873-880.

[17] Du, Z., Zhang, Y., Chen, Z., Li, P., Ni, Y., and Shi, L. 2012. Integrated emergency frequency control method for interconnected $\mathrm{AC} / \mathrm{DC}$ power systems using centre of inertia signals. IET Gener Transm Distrib 6, 6, pp. 584-592.

[18] Selvakumarana, S., Parthasarathy, S., Karthigaivel, R., and Rajasekaran, V. 2012. Optimal decentralized load frequency control in a parallel AC-DC interconnected power system through HVDC link using PSO algorithm. Energy Procedia 14, pp. 1849-1854. 